\title{
Antifungal agents commonly used in the superficial and mucosal candidiasis treatment: mode of action and resistance development
}

\author{
Małgorzata Bondaryk, Wiesław Kurzątkowski, Monika Staniszewska \\ National Institute of Public Health - National Institute of Hygiene, Warsaw, Poland \\ Head: Prof. Mirosław J. Wysocki
}

Postep Derm Alergol 2013; XXX, 5: 293-301

DOI: 10.5114/pdia.2013.38358

\begin{abstract}
Recent progress in medical sciences and therapy resulted in an increased number of immunocompromised individuals. Candida albicans is the leading opportunistic fungal pathogen causing infections in humans, ranging from superficial mucosal lesions to disseminated or bloodstream candidiasis. Superficial candidiasis not always presents a risk to the life of the infected host, however it significantly lowers the quality of life. Superficial Candida infections are difficult to treat and their frequency of occurrence is currently rising. To implement successful treatment doctors should be up to date with better understanding of $C$. albicans resistance mechanisms. Despite high frequency of Candida infections there is a limited number of antimycotics available for therapy. This review focuses on current understanding of the mode of action and resistance mechanisms to conventional and emerging antifungal agents for treatment of superficial and mucosal candidiasis.
\end{abstract}

Key words: antifungal agents, Candida albicans, mode of action, resistance mechanisms.

\section{Introduction}

Candida albicans infections are a problem of growing clinical importance worldwide. Literature data point out that this opportunistic pathogen is the leading cause of superficial and disseminated fungal infections in humans. Moreover, about $96 \%$ of all opportunistic mycoses are caused by Candida sp. [1-3]. In healthy individuals Candida colonizes mainly mucosal surfaces of the oral cavity, gastrointestinal and urogenital tracts without disease symptoms, where most frequently identified species are C. al-bicans (70\%) and C. glabrata (7\%) [3-7]. Moreover, an association between fungal colonization and candidiasis has been previously described. According to Nguyen et al. [8], colonization of the epithelial surfaces with pathogenic Candida strains is required for pathogenesis development. In immunocompromised humans, Candida frequently causes infections ranging from superficial mucosal lesions to disseminated or bloodstream infections [6]. More than 100 species of Candida have been identified, however only a few have been isolated from humans. Although C. albicans remains the most common cause of fungemia and hematogenously disseminated candidiasis, there has been an increase in infections caused by C. glabrata, C. tropicalis, C. parapsilosis, C. krusei, and C. lusitaniae [9]. Infections with these different species may require different therapeutic considerations [9]. Most human superficial and mucosal infections are caused by C. albicans, although other species such as C. glabrata, C. tropicalis, C. parapsilosis, C. kefyr, C. krusei, and C. gulliermondii may also be implicated in superficial as well as mucosal diseases. Although C. albicans can be cultured from the mouths of non-infected normal individuals, it does not cause oropharyngeal candidiasis unless predisposing factors exist to allow the infection to become established (Table 1) [3, 10, 11]. Candida albicans superficial infections include oral and vaginal thrush as well as chronic mucocutaneous candidiasis [12]. Being an important cause of morbidity and difficult to treat, superficial candidiasis of the mucosa, skin and nails have become a significant problem worldwide [11]. Although superficial candidiasis rarely presents a risk to the life of patients, it significantly lowers the quality of life $[11,13]$.

Address for correspondence: Monika Staniszewska PhD, Independent Laboratory of Streptomyces and Fungi Imperfecti, National Institute of Public Health - National Institute of Hygiene, 24 Chocimska St, 00-791 Warsaw, Poland, phone: +48 225421228 , fax: + 482284974 84, e-mail: mstaniszewska@pzh.gov.pl Received: 10.01.2013, accepted: 23.06.2013. 
Table 1. Risk factors for the development of oropharyngeal candidiasis

\begin{tabular}{|c|}
\hline Immunosupression \\
\hline HIV infection \\
\hline Chronic mucocutaneous candidiasis \\
\hline Neutropenia \\
\hline Drugs \\
\hline Cytotoxic chemiotherapy \\
\hline Corticosteroids \\
\hline Broad-spectrum antimicrobial agents \\
\hline Anticholinergics \\
\hline Diabetes mellitus \\
\hline Nutritional deficiencies \\
\hline Iron deficiency \\
\hline Malnutrition \\
\hline Prior or current local pathology \\
\hline Dentures \\
\hline Xerostomia \\
\hline Infancy \\
\hline
\end{tabular}

The high frequency of occurrence of candidiasis combined with difficult treatment cause a tendency toward better understanding of $C$. albicans resistance mechanisms. Therefore, this review focuses on characterization of antifungal agents commonly used in treatment and fungal resistance mechanisms. We discuss here several topical and systemic options for the treatment of candidiasis.

\section{Antifungal agents}

For the last three decades, fungal infections have become a major problem worldwide, especially among the immunocompromised individuals [3]. Despite that Candida is the leading cause of the opportunistic fungal infections, there is a limited number of antimycotics available for therapy [2, 3, 14]. Perea et al. [15] divided antifungal agents commonly used for candidiasis treatment in five major groups basing on their mode of action; group I: inhibition of RNA and/or DNA synthesis (fluorinated pyrimidine analogs 5-FC); group II: alteration of the membrane function (polyenes: nystatin, natamycin, amphotericin B AMB); group III: alteration of cell wall biosynthesis by inhibition of $\beta(1,3)$ glucan synthase (echinocandins: caspofungin, micafungin, anidulafungin); group IV: inhibition of ergosterol biosynthesis by inhibition of squalene epoxidase and/or accumulation of toxic sterol intermediates (allylamines: terbinafine, naftifine); and group $\mathrm{V}$ : inhibition of lanosterol demethylase in ergosterol biosynthesis (azoles) [14, 15].

\section{Fluorinated pyrimidine analog 5-FC}

Flucytosine (5-fluorocitosine, 5-FC) is a fluorinated derivative of the pyrimidine cytosine typically used in candidiasis treatment $[16,17]$. The antifungal activity of 5-fluorocitosine results from its rapid conversion to 5-fluorouracil [17]. Flucytosine is metabolized via the pyrimidine salvage pathway [18]. The 5-FC is taken up by cytosine permease (encoded by FCY2) and transported into fungal cells $[17,19]$. Next, this compound is enzymatically transferred into 5-fluorouracil (5-FU) by cytosine deaminase (encoded by FCY1) $[19,20]$. Moreover, the uracil phosphoribosyltransferase (encoded by FUR1) transforms 5-FU into 5-fluorouridine monophosphate (5-fluoro-UMP) [19]. The 5-fluoro-UMP inhibits DNA synthesis by targeting thymidylate synthetase. Furthermore, after transformation to 5-fluorouridine triphosphate (5-fluoro-UTP), this antifungal agent disturbs protein synthesis by incorporating into RNA, which results in the cell death $[16,20]$. Mammalian cells lack the cytosine deaminase enzyme, therefore they are not directly subject to the toxic effects of 5-FC [18]. By far, resistance to $5-F C$ is observed in vitro in $3-10 \%$ of $C$. albicans isolates. Moreover, $30 \%$ of isolates develop resistance during treatment with 5-FC [18, 19, 21]. According to literature data $[17,19]$, resistance to flucytosine is linked to the deficiency in enzymes involved in uptake, transport and transformations of 5-FC. Genes coding enzymes involved in 5-FC metabolism also contribute to cross resistance to flucytosine and fluconazole in Candida sp. [22]. Papon et al. [22] demonstrated that inactivation of FCY1, FCY2, FUR1 in C. lusitaniae produced two patterns of resistance, where mutant fur 1 was resistant to 5-fluorouracil, while mutants fcy 1 and $f c y 2$ were resistant to fluconazole. Moreover, fungal resistance might also result from an increased synthesis of pyrimidines that compete with fluorinated antimetabolites of 5-FC and therefore decrease its antifungal activity [17, 23]. As mentioned above, rapid development of resistance in Candida spp. during treatment with 5-FC was observed.

Therefore, the 5-FC monotherapy with few exceptions is not recommended. Moreover, toxic effects of 5-FC such as skin rash, nausea, bone marrow suppression, liver dysfunction, vomiting and diarrhea have been confirmed [24]. Despite that, flucytosine remains useful in multi-drug therapy in hematogenous candidiasis treatment as an adjunct to amphotericin B or azoles [21]. Furthermore, this antifungal agent is currently used in treatment of life-threatening Candida infections such as endocarditis, meningitis and hepatosplenic disease [18, 24].

\section{Polyenes}

Polyene antifungal agents are natural compounds derived from fermentation by Streptomyces [25]. Nystatin, natamycin and amphotericin B are three main polyene drugs used in mycoses treatment [26]. The parenteral administration of nystatin is followed by severe side effects [25]. Contrariwise, as this antifungal agent is not absorbed 
either from the gastrointestinal tract or via skin, both oral and topical administration of this antifungal agent are not related to significant toxic side effects [25]. Thus, nystatin is currently used in superficial candidiasis treatment, such as oral and vaginal infections with topic or oral administration only $[25,27]$. Superficial Candida infections of the skin can be treated topically with amphotericin B or nystatin. Congenital cutaneous candidiasis may resolve with topical and oral nystatin treatment. Nystatin creams used for treatment of an acute Candida vaginitis achieved mycological cure rates of approximately $75 \%$ to $80 \%$. On the other hand, polyenes (nystatin) achieved slightly lower clinical and mycological cure rates than azole agents [28] Natamycin is considered as a drug of choice in filamentous fungi topic treatment [29]. Moreover, this antifungal agent is currently used in fungal keratitis $[26,29]$.

Contrariwise to previous members of the polyene group, fungicidal in its nature amphotericin $B(A M B)$ was long considered as the gold standard in antifungal therapy [30, 31]. Currently, this antifungal agent is used in treatment of infections caused by such pathogens as Candida spp., Aspergillus spp., Fusarium spp., Cryptococcus spp., Mucor spp., Rhizopus spp., Trichosporon spp., Scedosporium spp. and Malassezia spp. [26, 30]. Despite the broad spectrum of antifungal activity, AMB is also characterized by widespread tissue distribution, long elimination half-life and a significant toxicity profile [32]. The toxicity profile of all polyenes result from their affinity for cholesterol which is a human counterpart for ergosterol [26]. The toxic side effects of AMB include infusion-related events, such as chills, fever, headache, nausea and vomiting, and dose-limiting nephrotoxicity [24].

The primary target of polyenes is ergosterol [14]. The AMB binds ergosterol localized in the fungal cell membrane leading to the pore formation, increased permeability of the membrane, oxidative damage and in the end leaking of cellular contents $[14,16]$. Although resistance to amphotericin $B$ is uncommon in C. albicans, other Candida species are thought to be intrinsically resistant to $A M B$ [16]. It has been estimated that decreased ergosterol content in the fungal cell membrane is associated with resistance to amphotericin $B$ as AMB-resistant Candida strains have relatively low ergosterol content in comparison to susceptible isolates [14, 33]. Lowering ergosterol content is associated with defects in the ERG3 gene, which leads to the accumulation of other sterols instead of ergosterol [19, 33-35].

Management of Candida superficial infections including treatments with amphotericin B. Low-dose amphotericin B $0.4 \mathrm{mg} / \mathrm{kg} /$ day is effective in the treatment of oropharyngeal candidiasis and esophageal candidiasis (if endoscopy confirms the persistence). Lipid formulation is available if the patient is unable to tolerate conventional amphotericin B. Duration of therapy is at least 10 to 14 days. The management of ocular infections consists of high-dose amphotericin B (0.7 to $1 \mathrm{mg} / \mathrm{kg} /$ day) preferably in conjunction with flucytosine, because of the poor intraocular pen- etration achieved by amphotericin B. Therapy should be continued for at least 10 to 14 days after resolution of all signs and symptoms of infection. So far, three lipid products of amphotericin B have been marketed: amphotericin B colloidal dispersion (ABCD, Amphocil), amphotericin $B$ lipid complex (ABLC, Abelcet), and liposomal amphotericin $B$ (L-AMB, AmBisome). Large prospective randomized trials showed that ABLC at a dose of $5 \mathrm{mg} / \mathrm{kg} /$ day was as effective as and less nephrotoxic than conventional amphotericin B at a dose of 0.7 to $1 \mathrm{mg} / \mathrm{kg} /$ day in hematogenous candidiasis. Overall, published data suggest that the three lipid formulations of amphotericin B are associated with less renal toxicity than conventional amphotericin B. Among the three lipid formulations of amphotericin B, L-AMB (AmBisome) is the least nephrotoxic and appears to result in significantly fewer infusion-related infections [36]. There is no consensus about appropriate dosing of the lipid formulations of amphotericin B. However, doses of L-AMB as low as 1 to $3 \mathrm{mg} / \mathrm{kg} /$ day and doses of $A B L C$ and $A B C D$ of $5 \mathrm{mg} / \mathrm{kg} /$ day seem to be adequate for treatment of Candida infections. Duration of therapy depends on the extent and seriousness of the infection. In view of the recent European study [37], most Candida strains were sensitive to amphotericin B. This is also good news for treatment of oral candidiasis as amphotericin B can be used topically in the form of lozenges.

\section{Echinocandins}

The echinocandins are a class of semisynthetic lipopeptide antifungal compounds synthetically derived from natural lipopeptides produced by Aspergillus rugulovalvus, Zalerion arboricola, Papularia sphaerosperma [26, 38]. While being fungicidal against $C$. albicans and fungistatic against Aspergillus spp. and despite high activity against Pneumocystis carinii, they have no effect on such pathogens as Cryptococcus, Trichosporon, Scedosporium, and Fusarium species [33, 39, 40]. Despite the narrow spectrum of activity, this group of antifungals is broadly effective against azole-resistant Candida strains [41]. Currently three members of this class are licensed for mycoses treatment: caspofungin (CAS), micafungin (MFG) and anidulafungin (ANI) $[16,42]$. Adverse events and toxic effects of echinocandins include headache, rash, fever, liver toxic effects, phlebitis, histamine release and hemolysis. However, their occurrence is rare [39]. The cell wall represents a perfect potential target for antifungal agents, as this structure is absent in mammalian cells [39]. Echinocandins disturb cell wall biosynthesis by inhibition of $1,3-\beta$-glucan synthase [14]. This enzyme is responsible for synthesis of $1,3-\beta$-glucan, which is a crucial component that strengthens the cell wall of C. albicans and S. cerevisiae [16]. Lack of the glucan component in the cell wall results in osmotic instability and ultimately in cell lysis [40]. Resistance to echinocandins is linked to a mutation in $1,3-\beta$-glucan synthase complex [14]. The $1,3-\beta$-glucan synthase consists of two units Rholp and Fksp, 
where Fksp is the active site of this enzyme and Rhol is the regulator $[39,42]$. Mutation in the FKS gene results in resistance to echinocandins $[14,42]$. It was previously described $[43,44]$ that mutations within the FKS gene of C. glabrata clinical isolate resulted in reduced susceptibility or resistance to echinocandins. Furthermore, fungal resistance might also result from lack of $1,3-\beta$-glucan in the fungal cell wall. It was described that the cell wall of $C$. neoformans consists mainly of $\alpha$-(1,3)- or $\alpha$-(1,6)-glucan and therefore, this species is resistant to the echinocandins [45]. By far, no cross-resistance with azoles or polyenes has been described, however mechanisms of resistance to echinocandins are still being investigated [33-35, 38, 39]. Caspofungin is as effective as amphotericin B for treatment of oropharyngeal candidiasis in doses of 50 to $70 \mathrm{mg} /$ day [36].

\section{Allylamines}

Terbinafine and naftifine are two main allylamines used in mycoses treatment. This class of antifungal compounds possess fungicidal activity against dermatophytes and fungistatic activity against $C$. albicans $[14,46]$. Terbinafine can be applied both systematically and topically and is widely used in treatment of fungal infections of skin, nails and hair $[47,48]$. The side effects of allylamines treatment are rare (2-3\% of patients) and include itching, burning and redness at the application site [46]. The case study of Ghannoum and Elewski [48] demonstrated that terbinafine used together with fluconazole provided successful treatment of oropharyngeal candidiasis which was not responding to fluconazole. Moreover, terbinafine demonstrated high fungicidal activity on itraconazole resistant fungi during in vitro study [49]. Allylamines inhibit ergosterol biosynthesis independent of cytochrome P-450 enzymes, by binding to squalene epoxidase (Erg1p) resulting in accumulation of squalene in high amounts inside the cell $[46,47,50]$. This leads to increased membrane permeability, disturbtion of cell organization and ultimately to cell death [46, 50]. According to Osborne et al. [51] and Cannon et al. [14], fungal resistance to terbinafine is due to a single amino acid substitution in Erg1p. In C. albicans, resistance to terbinafine might be also related to genes encoding membrane transport proteins CDR1, AGP2 and HOL3. Up-regulation of these genes serve to extrude the antifungal agent accumulated inside the cell out [52]. Moreover, according to Odds [53], resistance to terbinafine is linked to MAT locus homozygosity. The mentioned study [53] suggests that allylamines resistance is mediated by the same mechanism as efflux pump-mediate azole resistance. Other allylamines resistance mechanisms include induction of detoxification and stress tolerance [14]. Terbinafine seems to be more active against infections caused by C. parapsilosis and C. albicans. Topical antifungal agents may have a role in preventing relapse of the infections after successful oral therapy [36]. According to the latter authors [36], oral treatment options include terbinafine $250 \mathrm{mg} /$ week $\times 9$ to 18 months. Moreover, top- ical treatment onychomycosis due to Candida spp. are usually of little value.

\section{Azole antifungal agents}

Azole antifungal agents possess fungicidal activity against Aspergillus spp. and fungistatic activity against Candida spp. [54]. Several agents of this class including fluconazole (FLC), itraconazole (ITR), voriconazole (VRC) and posaconazole (POS) have been widely used in mycoses treatment [55]. Azoles target a lanosterol demethylase (Erg11p), a cytochrome P-450 enzyme mediating rate-limiting step in ergosterol biosynthesis [14, 19]. By binding the heme moiety in Erg11p, azoles inhibit activity of this enzyme and therefore disturb ergosterol biosynthesis [16]. Due to accumulated intermediates of ergosterol biosynthesis, a subsequent mechanism of sterol metabolism mediated by $C 5,6$ desaturase enzyme (encoded by ERG3) is activated $[34,56]$. The Erg3p mediates conversion of non-toxic $14 \alpha$ methylfecosterol to 3,6-diol (14amethylergosta-8,24(28) $-3 \beta, 6 \alpha$-diol) $[34,56]$. Conversion of Erg11p substrate into toxic methylated sterols leads to growth inhibition [14, 19]. Azole antifungal agents are mostly the first choice for antifungal therapy [57]. Yet, their fungistatic nature against Candida spp. caused a strong directional selection towards azole-resistant strains [54]. In a recent study, Ramesh et al. [58] tested the antifungal pattern of the C. albicans isolates from the oral cavity of HIV-infected patients. Results of the mentioned study [58] demonstrated growing resistance of $C$. albicans to tested azoles that varied from $11.9 \%$ to $41.1 \%$ depending on azole used. This causes a need for better understanding of fungal resistance mechanisms. The main azole resistance mechanisms in Candida and other pathogenic fungi include:

1) Mutations or overexpression of ERG11

Nonsynonymous mutations in ERG11 cause amino acid substitutions resulting in alteration of lanosterol demethylase and decreased azole affinity to this enzyme [55]. Moreover, each copy of ERG11 contributes to azole resistance [59]. Overexpression of ERG11 also compels the need for a higher azole concentration in order to bind all of Erg11p molecules present in the fungal cell [60].

2) Reduced accumulation of the azole inside fungal cell

a) Reduced uptake of azole. The study of Mansfield et al. [57] had proven that in C. albicans and other fungal pathogens, azoles are up-taken via energy-independent facilitated diffusion. Decreased azole up-take might be linked to changes in composition of fungal cell membrane [50]. Moreover, azole import levels vary among resistant $C$. albicans clinical isolates suggesting a role of import in resistance to azoles [57]. The data acquired by Mansfield et al. [57] suggest that all azoles use the same import mechanism mediated by a transporter, therefore a mutation in putative transporter would result in azole cross-resistance. 
b) Efflux via $A B C$ transporters. Lowering intracellular accumulation of azoles is energy dependent [57]. Two types of azole transporters in C. albicans have been described: the major superfamily transporter encoded by MDR1 and the ATP-binding cassette (ABC) transporters encoded by CDR1 and CDR2 [61]. The ABC transporters are ATP-dependent whereas Mdr1p depends on utilization of the proton motive force at the cellular membrane in order to transport azoles out of the cell [57]. Overexpression of these drug efflux pumps transport azoles out of the cell, therefore reducing intracellular drug accumulation [50, 62]. Upregulation of $M D R 1$ results in fluconazole resistance, contrariwise upregulation of $A B C$ transporters leads to multi azole resistance [63]. The expression of drug efflux pumps is regulated by TAC1 and MRR1 [55]. According to Coste et al. [63], Candida strains that are homozygous at the mating-type locus and have hyperactive TAC1 possess an increased resistance to fluconazole. Hyperactive TAC1 causes a constitutive high expression of CDR1 and CDR2 [63]. Yet, the loss of heterozygosity $(\mathrm{LOH})$ also fulfills an important role in the azole resistance mechanism as TAC hyperactive phenotype occurs only when it is homozygous $[59,63]$. Moreover, according to Hoot et al. [64], in C. albicans $\mathrm{LOH}$ occurs through a recombinationmediated event via homologous recombination pathways. However, defects in homologous recombination might result in altered $\mathrm{LOH}$ events and therefore sensibility to azoles [64].

3) Tolerance to methylated sterols via mutation in ERG3 A defective Erg3p function resulting in changed membrane sterol content has been linked to azole resistance $[34,62]$. It was documented [35] that azole-resistant $C$. albicans isolates exhibit defective sterol $\Delta^{5,6}$-desaturation. Mutation in sterol $\Delta^{5,6}$-desaturase results in production of $14 \alpha$-methylfecosterol which is capable of cell growth [35]. Cells with defective sterol $\Delta^{5,6}$-desaturation lack ergosterol (as Erg11p is still inhibited by azole drug) and therefore are cross-resistant to polyenes such as amphotericin $B[34,35]$. It was previously suggested [65] that mutations in filamentation regulator EFG1 increase susceptibility to azoles as this gene participates in regulating the expression of ERG3.

4) Biofilm formation

Candida biofilms are composed of cells embedded in an extracellular matrix [66]. Those structures are highly resistant to antifungal treatment, especially to azoles and AMB $[54,66]$. Resistance of biofilms to azole drugs results from conjunction of several mechanisms acting in a time-dependent manner, including such phenomena as phenotype changes due to decreased growth or/and nutrient limitation; surface-induced expression of the resistance genes; disabled drug penetration through biofilm matrix; high cell density; decreased ergosterol levels in mature biofilms; upregulation of genes coding drug efflux pumps during biofilm formation; presence of highly azole-resistant persister cells in the biofilm structure [66-68]. Glucan present in extracellular matrix sequesters azoles and prevents them from reaching the target enzyme [54]. Therefore, Candida biofilms remain sensitive to the newly introduced echinocandins that target cell wall $\beta$-glucan biosynthesis [66]. In C. albicans matrix, glucan levels are regulated by the molecular chaperone Hsp90 - the heat shock protein 90. By compromising its function, biofilm dispersal is blocked and its ability to serve as potential source of infection is reduced [54].

5) Import of host cholesterol

In the absence of ergosterol, some fungal pathogens such as Candida glabrata and Aspergillus fumigatus are able to import and utilize host cholesterol [69-71]. This phenomenon was also described in Saccharomyces cerevisiae $[72,73]$. Fungi incorporate exogenous sterols from serum under aerobic (C. glabrata and $A$. fumigatus) or anaerobic (S. cerevisiae) conditions [70, 71, 73]. According to Xiong et al. [71], accelerated growth along with the extensive cholesterol import occurs in the presence of serum. Moreover, it was demonstrated [71] that import cholesterol uptake significantly increases in the presence of azoles and it appears to attenuate effects of these antifungal agents. The results of previous studies $[70,71,73]$ suggested that incorporation of host cholesterol by fungi complements defects in ergosterol biosynthesis, therefore suppressing growth defects and azole toxicity.

Treatments for superficial candidiasis are fluconazole, itraconazole, and ketoconazole [74]. These are generally used for severe or chronic oral candidiasis and chronic mucocutaneous candidiasis. The daily doses used are ketoconazole $200 \mathrm{mg}$ (400 mg in AIDS patients), itraconazole, and ketoconazole. According to Horgan and Powderly [10], treatment of oropharyngeal candidiasis is relatively simple, with most types responding well to therapy. Generally, the latter authors [10] discussed that clotrimazole, ketoconazole, fluconazole, and itraconazole are probably equivalent in the acute treatment of most cases of oropharyngeal candidiasis. The duration of therapy is also variable and in uncomplicated infections there has been tendency to shorten the course of therapy. As reported Horgan and Powderly [10], patients should receive itraconazole and fluconazole for at least 7 days. It was described that daily fluconazole was more effective than clotrimazole in preventing mucosal candidiasis. Ketoconazole and itraconazole are probably also useful but have not been extensively evaluated in controlled trials. Moreover, fluconazole at dosages $50 \mathrm{mg} /$ day to $400 \mathrm{mg}$ /day has been effective in preventing oropharyngeal candidiasis and decreasing colonization with Candida. On the other hand, because fluconazole is less active against $C$. glabrata and C. krusei, increased colonization and, at some centers, increased infections with these species has been reported when fluconazole was used routinely for prophylaxis. In patients with infection caused by organisms 
with intermediate susceptibility, higher doses of fluconazole (up to $800 \mathrm{mg}$ ) may be tried. Moreover, other azoles may be efficacious because some fluconazole-resistant isolates retain sensitivity to itraconazole and ketoconazole. Resistance to fluconazole or ketoconazole may develop if the drug is used continuously in the face of clinically unresponsive infection. Vaginal infections respond to intensive topical therapy given for 3 to 5 days with either cream or vaginal tablets. In these cases, nystatin or an imidazole such as miconazole is used. Chronic or persistent infection is a clinical problem that does not have a reproducible solution. However, it is important in all such cases to ensure by way of cultures that symptoms are caused by Candida and not other infectious agents. Regimens that have been attempted include continuous fluconazole or itraconazole for 1 to 2 months followed by intermittent midcycle therapy with either itraconazole $400 \mathrm{mg}$ daily for 2 to 3 days or fluconazole $200 \mathrm{mg}$ daily for a similar period. Despite these measures, relapse is common. For infec- tions of the skin surface, azole creams or ointments are usually successful [10]. In the case of patients with acute vaginal Candida infections, azole agents achieved clinical and mycological cure rates of approximately $85 \%$ to $90 \%$. Contrariwise, there is little evidence that any azole agent is superior to others. Oral antimycotic agents i.e., ketoconazole (400 mg daily for 5 days), itraconazole (200 mg daily for 3 days or 400 mg for 1 day), and fluconazole (150 mg in a single daily dose) were all shown to be highly effective in achieving clinical mycological cure in acute Candida vaginitis. Management of Candida vulvovaginitis during pregnancy is more difficult, because clinical response tends to be slower and recurrences are more frequent. Sobel [28] reported that most topical antifungal agents are effective, especially when prescribed for longer periods of 1 to 2 weeks, however, single-dose therapy with clotrimazole was shown to be effective during pregnancy. The management of women with recurrent vulvovaginal candidiasis RVVC (defined as more than 4 epi-

Table 2. Therapy for vaginal candidiasis

\begin{tabular}{|c|c|c|}
\hline Drug & Formulation & Dosage regimen \\
\hline \multicolumn{3}{|c|}{ Topical agents } \\
\hline Butoconazole & $2 \%$ cream & $5 \mathrm{~g}$ for 3 days \\
\hline \multirow[t]{4}{*}{ Clotrimazole } & $1 \%$ cream & 5 g for $7-14$ days \\
\hline & 100 mg vaginal tablets & 1 tablet for 7 days \\
\hline & 100 mg vaginal tablets & 2 tablets for 3 days \\
\hline & 500 mg vaginal tablets & 1 tablet - single dose \\
\hline \multirow[t]{4}{*}{ Miconazole } & $2 \%$ cream & $5 \mathrm{~g}$ for 7 days \\
\hline & 100 mg vaginal suppository & 1 suppository for 7 days \\
\hline & 200 mg vaginal suppository & 1 suppository for 3 days \\
\hline & 1200 mg vaginal suppository & 1 suppository - single dose \\
\hline Econazole & 150 mg vaginal tablets & 1 tablet for 3 days \\
\hline Fenticonazole & $2 \%$ cream & $5 \mathrm{~g}$ for 7 days \\
\hline \multirow[t]{2}{*}{ Tioconazole } & $2 \%$ cream & $5 \mathrm{~g}$ for 3 days \\
\hline & $6.5 \%$ cream & $5 \mathrm{~g}-$ single dose \\
\hline \multirow[t]{3}{*}{ Terconazole } & $0.4 \%$ cream & $5 \mathrm{~g}$ for 7 days \\
\hline & $0.8 \%$ cream & $5 \mathrm{~g}$ for 7 days \\
\hline & 80 mg vaginal suppository & $80 \mathrm{mg}$ for 3 days \\
\hline Nystatin & $100000 \mathrm{U}$ vaginal tablets & 1 tablet for 14 days \\
\hline \multicolumn{3}{|c|}{ Oral agents } \\
\hline Ketoconazole & $400 \mathrm{mg}$ bid & for 5 days \\
\hline \multirow[t]{2}{*}{ Intraconazole } & $200 \mathrm{mg}$ bid & for 1 day \\
\hline & $200 \mathrm{mg}$ & for 3 days \\
\hline Fluconazole & $150 \mathrm{mg}$ & Single dose \\
\hline
\end{tabular}


sodes per year) requires systemic azoles fluconazole or itraconazole for 14 days, followed by a maintenance regimen (fluconazole 150 or 100 mg weekly for 6 months or daily low-dose ketoconazole - 100 mg daily for 6 months is well tolerated and efficacious) $[36,75,76]$. As an alternative to daily ketoconazole, weekly therapy with oral fluconazole $(100 \mathrm{mg})$ or topical clotrimazole $(500 \mathrm{mg})$ can be used. Treating RVVC remains challenging; long-term prophylaxis with 150 mg fluconazole once weekly for 6 months resulted in $91 \%$ of relapse-free patients at the end of treatment, but symptomatic relapse occurred in $57 \%$ of patients within 6 months after the cessation of treatment [77]. As described by Sobel [28], in patients with frequent recurrence of $C$. glabrata after an initial response to the aforementioned agents, a long-term regimen of topical nystatin in combination with ketoconazole or itraconazole can be prescribed after in vitro susceptibility tests indicate azole susceptibility. Reversely to acute Candida vaginitis, antimycotics available for local use as creams, lotions, aerosol sprays, vaginal tablets, suppositories, and coated tampons are presented in Table 2.

\section{Conclusions}

Rapidly growing resistance of fungal pathogens to commonly used antifungal agents remains a concern for modern medicine. Most of Candida resistance mechanisms result from point mutations of target enzymes or regulatory genes. Moreover, a broad use of antimycotics caused a directional selection among targeted pathogenic populations towards those with effective resistance mechanisms. Emergence of resistant strains resulted in an increased mortality rate and therefore compelled the need for search for novel antimycotics and new potential drug targets. When Candida strains show reduced susceptibility to antifungal drugs [78], it is imperative to keep in mind the need for careful screening of drug resistance of Candida isolates among non- and hospitalized patients and this should be considered carefully by clinicians. In the treatment of mycotic diseases, detailed in vitro and in vivo studies are needed to specify the extent of their effectiveness. Furthermore, viewpoints of the most recent data $[79,80]$ on oral and vaginal candidiasis therapy explored the treatment with probiotic bacteria that may be an effective alternative to prevent it. In this context, further studies are needed to evaluate the promising colonization results of these studies.

\section{Acknowledgments}

This work was supported by the National Science Centre, project DEC-2011/03/D/NZ7/06198.

\section{References}

1. Kourkoumpetis T, Manolakaki D, Velmahos GC, et al. Candida infection and colonization among non-trauma emergency surgery patients. Virulence 2010; 1: 359-66.
2. Naglik JR, Challacombe SJ, Hube B. Candida albicans secreted aspartyl proteinases in virulence and pathogenesis. Microbiol Mol Biol Rev 2003; 67: 400-28.

3. Pfaller MA, Diakema DJ. Epidemiology of invasive candidiasis: a persistent public health problem. Clin Microbiol Rev 2007; 20: 133-63.

4. Gropp K, Schild L, Schindler S, et al. The yeast Candida albicans evades human complement attack by secretion of aspartyl proteases. Mol Immunol 2009; 47: 465-75.

5. Soll DR, Lockhart SR, Zhao R. Relationship between switching and mating in Candida albicans. Eukaryot Cell 2002; 2: 390-7.

6. Szabo EK, MacCallum DM. The contribution of mouse models to our understanding of systemic candidiasis. FEMS Microbiol Lett 2011; 320: 1-8.

7. Lacour M, Zunder T, Huber R, et al. The pathogenetic significance of intestinal Candida colonization: a systematic review from an interdisciplinary and environmental medical point of view. Int J Hyg Health 2002; 205: 257-68.

8. Nguyen KA, Zmeter G, Claris O, Kassai B. Epidemiology of invasive candidiasis in neonatal invasive care unit in France. Acta Paediatr 2011; 101: e137-9.

9. Anaissie EJ, McGinnis MR, Pfaller MA. Clinical mycology. Elsevier Science, Churchill Livingstone, USA 2003.

10. Horgan MM, Powderly WG. Oral fungal infections. In: Clinical mycology. Anaissie EJ, McGinnis MR, Pfaller MA (eds.). Elsevier Science, Churchill Livingstone 2003; 443-55.

11. Borges M, Degreef H, Cauwenbergh G. Fungal infections of the skin: infection process and antimycotic therapy. Curr Drug Targets 2005; 6: 849-62.

12. Molero G, Diez-Orejas R, Navarro-Garcia F, et al. Candida albicans: genetics, dimorphism and pathogenicity. Interantl Microbiol 1998; 1: 95-106.

13. Rotta I, Otuki MF, Sanches ACC, Correr CJ. Efficacy of topical antifungal drugs in different dermatomycoses: a systemic review with meta-analysis. Rev Assoc Med Bras 2012; 58 : 303-13.

14. Cannon RD, Lamping E, Holmes AR, et al. Efflux-mediated antifungal drug resistance. Clin Microbiol Rev 2009; 22: 291-321.

15. Perea S, Patterson TF. Antifungal resistance in pathogenic fungi. Clin Infect Dis 2002; 35: 1073-80.

16. Barker KS, Rogers PD. Recent insights into the mechanism of antifungal resistance. Curr Infect Dis Rep 2006; 8: 449-56.

17. Vermes A, Guchelaar HJ, Dankert J. Flucytosine: a review of its pharmacology, clinical indications, pharmacokinetics, toxicity and drug interactions. I Antimicrob Chemother 2000; 46: 171-9.

18. Hope WW, Tabernero L, Denning DW, Anderson MJ. Molecular mechanisms of primary resistance to flucytosine in Candida albicans. Antimicrob Agents Chemother 2004; 48: 4377-86.

19. Espinel-Ingroff A. Mechanisms of resistance to antifungal agents: yeasts and filamentous fungi. Rev Iberoam Micol 2008; 25: 101-6.

20. Abaci O, Haliki-Uztan A. Investigation of susceptibility of Candida species isolated from denture wearers to different antifungal antibiotics. Afr J Microbiol Res 2011; 5: 1398-403.

21. Pfaller MA, Messer SA, Boyken L, et al. In vitro activities of 5 -fluorocitosine against 8,803 clinical isolates of Candida sp.: global assessment of primary resistance using national committee for clinical laboratory standards susceptibility testing methods. Antimicrob Agents Chemother 2002; 46: 3518-21. 
22. Papon N, Noël T, Florent M, et al. Molecular mechanism of flucytosine resistance in Candida lusitaniae: contribution of the FCY2, FCY1 and FUR1 genes to 5-fluorouracil and fluconazole cross-resistance. Antimicrob Agents Chemother 2007; 51: 369-71.

23. Polak A. 5-fluorocitosine-current status with special reference to mode of action and drug resistance. Contrib Microbiol Immunol 1977; 4: 158-67.

24. Dismukes WE. Introduction to antifungal drugs. Clin Infect Dis 2000; 30: 653-7.

25. Larson JL, Wallace TL, Tyl RW, et al. The reproductive and developmental toxicity of the antifungal drug Nyotran (liposomal nystatin) in rats and rabbits. Toxicol Sci 2000; 53: 421-9.

26. Vandeputte P, Ferrari S, Coste AT. Antifungal resistance and new strategies to control fungal infections. Int J Microbiol 2012; 2012: 713687.

27. Martins HPR, Da Silva MC, Paiva LCF, et al. Efficacy of fluconazole and nystatin in the treatment of vaginal Candida species. Acta Derm Venereol 2012; 92: 78-82.

28. Sobel JD. Fungal infections of the genitourinary tract. In: Clinical mycology. Anaissie EJ, McGinnis MR, Pfaller MA (eds.). Elsevier Science, Churchill Livingstone 2003; 496-508.

29. Srinivasan M. Fungal keratitis. Curr Opin Ophthalmol 2004; 15: 321-7.

30. Ellis D. Amphotericin B: spectrum and resistance. J Antimicrob Chemother 2002; 49 (Suppl. 1): 7-10.

31. Kumar R, Shukla PK. Amphotericin B resistance leads to enhanced proteinase and phospholipase activity and reduced germ tube formation in Candida albicans. Fungal Biol 2010; 114: 189-97.

32. Bartell A, Phatak A, Horn K, Postelnick M. Drug interactions involving antifungal drugs: time course and clinical significance. Curr Fungal Infect Rep 2010; 4: 103-10.

33. Kanafani ZA, Perfect JR. Resistance to antifungal agents: mechanisms and clinical impact. CID 2008; 46: 120-8.

34. Kelly SL, Lamb DC, Kelly DE, et al. Resistance to fluconazole and amphotericin in Candida albicans from AIDS patients. Lancet 1996; 348: 1523-4.

35. Kelly SL, Lamb DC, Kelly DE, et al. Resistance to fluconazole and cross-resistance to amphotericin B in Candida albicans from AIDS patients caused by defective sterol delta5,6-desaturation. FEBS Lett 1997; 400: 80-2.

36. Dignani MC, Solomkin JS, Anaissie EJ. Candida. In: Clinical mycology. Anaissie EJ, McGinnis MR, Pfaller MA (eds.). Elsevier Science, Churchill Livingstone 2003; 195-239.

37. Lass-Flörl C, Mayr A, Perkhofer S, Hinterberger G. Activities of antifungal agents against yeast and filamentous fungi: assessment according to the methodology of the European Committee on antimicrobial susceptibility testing. Antimicrob Agents Chemother 2008; 52: 3637-41.

38. Petraitiene R, Petraitis V, Groll AH, et al. Antifungal activity of LY303366, a novel echinocandin B, in experimental disseminated candidiasis in rabbits. Antimicrob Agents Chemother 1999; 43: 2148-55.

39. Denning DW. Echinocandin antifungal drugs. Lancet 2003; 362: 1142-51.

40. Ripeau JS, Aumont F, Belhumeur P, et al. Effect of echinocandin caspafungin on expression of Candida albicans secretory aspartyl proteinases and phospholipase in vitro. Antimicrob Agents Chemother 2002; 46: 3096-100.

41. Chen SA, Playford EG, Sorrell TC. Antifungal therapy in invasive fungal infections. Curr Opin Pharmacol 2010; 10: 1-9.

42. Bal AM. The echinocandins: three useful choices or three too many? Int J Antimicrob Agents 2010; 35: 13-8.
43. Thompson 3rd GR, Wiederhold NP, Vallor AC, et al. Development of Caspofungin resistance following prolonged therapy for invasive candidiasis secondary to Candida glabrata infection. Antimicrob Agents Chemother 2008; 52: 3783-5.

44. Tscherner M, Schwarzmüller T, Kuchler K. Pathogenesis and antifungal drug resistance of the human fungal pathogen Candida glabrata. Pharmaceuticals 2011; 4: 169-86.

45. Petrikkos G, Skiada A. Recent advances in antifungal chemotherapy. Int J Antimicrob Agents 2007; 30: 108-17.

46. Brennan B, Leyden JJ. Overview of topical therapy for common superficial fungal infections and the role of new topical agents. J Am Acad Dermatol 1997; 36: S3-5.

47. Gauwerky K, Borelli C, Korting HC. Targeting virulence: a new paradigm for antyfungals. Drug Discov Today 2009; 14: 214-22.

48. Ghannoum MA, Elewski B. Successful treatment of fluconazole-resistant oropharyngeal Candidiasis by a combination of fluconazole and terbinafine. Clin Diagn Lab Immunol 1999; 6: 921-3.

49. Hazen KC. Fungicidal versus fungistatic activity of terbinafine and intraconazole: an in vitro comparison. JAAD 1998; 38: S37-41.

50. Van Minnebruggen G, François IEJA, Cammue BPA, et al. A general overview on past, present and future antimicotics. Open Mycol J 2010; 4: 22-32.

51. Osborne CS, Leitner I, Hofbauer B, et al. Biological, biochemical, and molecular characterization of a new clinical Trichophyton rubrum isolate resistant to terbinafine. Antimicrob Agents Chemother 2006; 50: 2234-6.

52. Zeng Y, Qian Y, Ma L, Gu H. Genome-wide expression profiling of the response to terbinafine in Candida albicans using a cDNA microarray analysis. Chin Med I 2007; 120: 807-13.

53. Odds FC. In Candida albicans, resistance to flucytosine and terbinafine is linked to MAT locus homozygosity and multilocus sequence typing clade 1. FEMS Yeast Res 2009; 9: 1091-101.

54. Robbins N, Uppuluri P, Nett J, et al. Hsp90 governs dispersion and drug resistance of fungal biofilms. PLoS Pathog 2011; 7: e1002257.

55. MacCallum DM, Coste A, Ischer F, et al. Genetic Dissection of azole resistance mechanisms in Candida albicans and their validation in mouse model of disseminated infection. Antimicrob Agents Chemother 2010; 54: 1476-83.

56. Vale-Silva LA, Coste AT, Ischer $F$, et al. Azole resistance by loss of the sterol delta5,6-desaturase gene (ERG3) in Candida albicans does not necessarily decrease virulence. Antimicrob Agent Chemother 2012; 56: 1960-8.

57. Mansfield BE, Oltean HN, Oliver BG, et al. Azole drugs are imported by felicitated diffusion in Candida albicans and other pathogenic fungi. PLoS Pathog 2010; 6: e1001126.

58. Ramesh N, Priyadharsini M, Sumathi CS, et al. Virulence factors and antifungal sensivity pattern of Candida sp. isolated from HIV and TB patients. Indian J Microbiol 2011; 51: 273278.

59. Selmecki A, Geraml-Nejad M, Paulson C, et al. An isochromosome confers drug resistance in vivo by application of two genes, ERG11 and TAC1. Mol Microbiol 2008; 68: 624-41.

60. Perea S, López-Ribot JL, Kirkpatrick WR, et al. Prevalence of molecular mechanisms of resistance to azole antifungal agents in Candida albicans strains displaying high-level fluconazole resistance isolated from human immunodeficiency virus-infected patients. Antimicrob Agents Chemother 2001; 45: 2676-84. 
61. Vermitsky JP, Edlind TD. Azole resistance in Candida glabrata: coordinate upregulation of multidrug transporters and evidence for Prd1-like transcription factor. Antimicrob Agent Chemother 2004; 48: 3373-81.

62. Yan L, Li M, Cao Y, et al. The alternative oxidase of Candida albicans causes reduced fluconazole susceptibility. J Antimicrob Chemother 2010; 64: 764-73.

63. Coste A, Turner V, Ischer F, et al. A mutation in Tac1p, a transcription factor regulating CDR1 and CDR2, is coupled with loss of heterozigosity at chromosome 5 to mediate antifungal resistance in Candida albicans. Genetics 2006; 172: 2139-56.

64. Hoot SJ, Zheng X, Potensky CJ, et al. The role of Candida albicans homologous recombination factors Rad54 and Rdh54 in DNA damage sensitivity. BMC Microbiol 2011; 11: 214.

65. Lo HJ, Wang JS, Lin CY, et al. Efg1 involved in drug resistance by regulating the expression of ERG3 in Candida albicans. Antimicrob Agents Chemother 2005; 49: 1213-5.

66. d'Enferd C. Biofilms and their role in the resistance of pathogenic Candida to antifungal agents. Curr Drug Targets 2006; 7: 465-70.

67. Douglas JL. Candida biofilms and their role in infection. TRENDS Microbiol 2003; 11: 30-6.

68. Ramage G, Rajendran R, Sherry L, Williams C. Fungal biofilm resistance. Int J Microbiol 2012; 2012: 528521.

69. Becker JM, Kauffmann SJ, Hauser M, et al. Pathway analysis of Candida albicans survival and virulence determinants in a murine infection model. Proc Natl Acad Sci U S A 2010; 107: 22044-9.

70. Nakayama H, Izuta M, Nakayama N, et al. Depletion of squalene synthase (ERG9) gene does not impair growth of Candida grabata in mice. Antimicrob Agents Chemother 2000; 44: 2411-8.

71. Xiong Q, Hassan SA, Wilson WK, et al. Cholesterol import by Aspergillus fumigatus and its influence on antifungal potency of sterol biosynthesis inhibitors. Antimicrob Agent Chemother 2005; 49: 518-24.

72. Barns SM, Lane DJ, Sogin ML, et al. Evolutionary relationships among pathogenic Candida species and relatives. J Bacteriol 1991; 173: 2250-5.

73. Nakayama H, Tanabe K, Bard M, et al. The Candida grabata putative sterol transporter gene CgAUS1 protects cells against azoles in the presence of serum. J Antimicrob Chemother 2007; 60: 1264-72.

74. Hay RJ. Cutaneous and subcutaneous mycoses. In: Clinical mycology. Anaissie EJ, McGinnis MR, Pfaller MA (eds.). Elsevier Science. Churchill Livingstone 2003; 456-73.

75. Shahid Z, Sobel JD. Reduced fluconazol susceptibility of Candida albicans isolates in women with recurrent vulvovaginal candidiasis: effects of long-term fluconazole therapy. Diagn Microbiol Infect Dis 2009; 64: 354-6.

76. Bulik CC, Sobel JD, Nailon MD. Susceptibility profile of vaginal isolates of Candida albicans prior to and following fluconazole introduction - impact of two decades. Mycoses 2011; 54: 34-8.

77. Beikert FC, Le MT, Koeninger A, et al. Recurrent vulvovaginal candidosis: focus on vulva. Mycoses 2011; 54: e807-10.

78. Meurman JH, Pärnänen P, Seneviratne CJ, et al. Prevalence and antifungal drug sensitivity of non-albicans Candida in oral rinse samples of self-caring elderly. Gerodontology 2011; 28: 246-52.

79. Matsubara VH, Silva EG, Paula CR, et al. Treatment with probiotics in experimental oral colonization by Candida albicans in murine model (DBA/2). Oral Dis 2012; 18: 260-4.
80. Ehrström S, Daroczy K, Rylander E, et al. Lactic acid bacteria colonization and clinical outcome after probiotic supplementation in conventionally treated bacterial vaginosis and vulvovaginal candidiasis. Microbes Infect 2010; 12: 691-9. 